\title{
MEDIDA INDIRETA DA CLOROFILA E SUA RELAÇÃO COM O MANEJO DA ADUBAÇÃO NITROGENADA EM PLANTAS CÍTRICAS FERTIRRIGADAS ${ }^{1}$
}

\author{
THAIS REGINA DE SOUZA², LEANDRO CAIXETA SALOMÃO ${ }^{3}$, \\ THOMAS FIORE DE ANDRADE ${ }^{4}$, ROBERTO LYRA VILLAS BÔAS 5 , JOSÉ ANTÔNIO QUAGGIO 6
}

RESUMO - O planejamento da programação nutricional na citricultura requer a avaliação da disponibilidade de nutrientes através de análises de solo e folha, e também leva em consideração a expectativa de produtividade e a exportação de nutrientes pela colheita. A disponibilidade de nitrogênio $(\mathrm{N})$ não tem sido eficientemente avaliada através de análises do solo e, em algumas áreas de cultivo de plantas cítricas, o $\mathrm{N}$-foliar vem sendo empregado como guia para recomendação de N. O objetivo deste estudo foi avaliar a sensibilidade da medida indireta da clorofila como um método de monitoramento dos níveis de $\mathrm{N}$ em plantas cítricas. Para isso, um trabalho experimental de campo foi conduzido em Reginópolis-SP, durante dois anos, com as variedades de copa Valência e Hamlim, ambas sobre porta-enxerto citrumelo Swingle. Os tratamentos consistiram em cinco doses de $\mathrm{N}$ aplicadas via fertirrigação: $0 ; 35 ; 70 ; 140$ e $280 \mathrm{~kg} \mathrm{ha}^{-1}$ de $\mathrm{N}$, na forma de nitrato de amônio. Todas as parcelas foram devidamente fertilizadas com fósforo, potássio e micronutrientes também através da fertirrigação. A medida indireta da clorofila foi avaliada pelo clorofilômetro, modelo SPAD-502, em folhas recém-maduras ( $3^{\mathrm{a}}$ e $4^{\mathrm{a}}$ folhas), nos quatro quadrantes, e na altura mediana da planta. As mesmas folhas foram utilizadas para a determinação do teor de $\mathrm{N}$-foliar. As medidas de leitura SPAD e o teor de $\mathrm{N}$ na folha apresentaram respostas quadráticas às doses de $\mathrm{N}$ aplicadas. Correlação de $0,95(\mathrm{p}<0,05)$ foi observada entre a medida indireta da clorofila e o teor de $\mathrm{N}$ foliar. Durante os estádios de desenvolvimento da cultura, os valores médios de leitura SPAD variaram em 11 unidades, e os teores médios de $\mathrm{N}$ foliar, em 5 unidades $\left(\mathrm{g} \mathrm{kg}^{-1}\right)$. Leituras SPAD abaixo de 70 e acima de 75 estiveram relacionadas, respectivamente, com plantas muito responsivas e não responsivas a $\mathrm{N}$, enquanto plantas com leituras intermediárias devem ser adubadas com doses de $\mathrm{N}$ próximas daquelas extraídas com a colheita. Baseado em duas safras completas, conclui-se que a medida indireta da clorofila pode ser empregada como ferramenta rápida e não destrutiva no monitoramento e na avaliação da disponibilidade de $\mathrm{N}$ em plantas cítricas

Termos para indexação: citros, clorofilômetro, teor foliar, disponibilidade de nitrogênio.

\section{CHLOROPHYL INDIRECT MEASUREMENT AND ITS RELATIONSHIP WITH THE NITROGEN FERTILIZATION MANAGEMENT IN FERTIGATED CITRUS PLANTS}

\begin{abstract}
The planning of nutritional programming in citrus requires the evaluation of nutrient availability through soil and leaf analysis and also considers the yield expectancy and the nutrient exportation by the harvest. Nitrogen $(\mathrm{N})$ availability has not being efficiently evaluated by soil analysis and for this reason leaf- $\mathrm{N}$ has being used as a guide to $\mathrm{N}$ recommendation in some areas with citrus growers. This study aimed to evaluate the sensitivity of the chlorophyll indirect measurement as a method to monitor $\mathrm{N}$ status of citrus trees. A field trial was conducted in Reginópolis, State of São Paulo - Brazil, during two consecutive years, with Valencia and Hamlin trees, both grafted on citrumelo Swingle rootstock. The treatments consisted of five nitrogen rates applied by fertigation: $0,35,70,140$ and $280 \mathrm{~kg} \mathrm{ha}^{-1}$ of $\mathrm{N}$ as ammonium nitrate. All plots were adequately supplied with phosphorus, potassium and micronutrients also by fertigation. The chlorophyll indirect measurement was evaluated through the SPAD-502 chlorophyll meter in recent mature leaves $\left(3^{\text {th }}\right.$ and the $4^{\text {th }}$ leaves), in the four tree quadrants at the medium of tree height. The same leaves were used to assess leaf $\mathrm{N}$ concentration. SPAD readings and leaf $\mathrm{N}$ concentrations showed quadratic trends when plotted against $\mathrm{N}$ rates. A correlation of $0.95(\mathrm{p}<0.05)$ was observed between the chlorophyll indirect measurement and leaf $\mathrm{N}$ concentration. During the different tree development stages, SPAD readings varied by 11 units, while leaf-N variation was $5 \mathrm{~g} \mathrm{~kg}^{-1}$. SPAD readings below 70 and above 75 were respectively related to very $\mathrm{N}$ responsive and non $\mathrm{N}$ responsive trees, while trees with intermediate reading values should be supplied with $\mathrm{N}$ rates close to those removed by harvest. Based on the results of two successive seasons, it is concluded that the chlorophyll indirect measurement can be used as a fast and non-destructive tool to monitor and to assess nitrogen availability in citrus plants.

Index terms: citrus, chlorophyll meter, leaf-N, nitrogen availability.

\footnotetext{
1(Trabalho 230-10). Recebido em: 25-10-2010. Aceito para publicação em: 15-02-2011. Trabalho extraído de Tese de Doutorado. Suporte financeiro Fapesp.

${ }^{2}$ Pós-Doutoranda do IAC, Centro de Solos e Recursos Agroambientais. Campinas-SP, E-mail: tr_souza@yahoo.com.br ${ }^{3}$ Doutorando do Curso de Pós-Graduação em Irrigação e Drenagem, FCA/UNESP. Botucatu-SP, E-mail: 1salomao@fca.unesp.br ${ }^{4}$ Mestrando do Curso de Pós-Graduação em Irrigação e Drenagem, FCA/UNESP, E-mail: tfandrade@fca.unesp.br ${ }_{5}^{5}$ Professor Doutor do Departamento de Recursos Naturais/Ciência do Solo, FCA/UNESP, E-mail: rlvboas@fca.unesp.br
} ${ }^{6}$ Pesquisador do IAC, Centro de Solos e Recursos Agroambientais, E-mail: quaggio@iac.sp.gov.br
\end{abstract}




\section{INTRODUÇÃO}

$\mathrm{Na}$ citricultura paulista, a irrigação já ocupa aproximadamente $21 \%$ da área cultivada, o que corresponde a aproximadamente 125 mil hectares (COELHO, 2010). Trata-se de investimento relativamente alto, que foi motivado pelas condições climáticas desfavoráveis dos últimos anos e pela mudança do porta-enxerto limão-Cravo, suscetível à morte súbita dos citros. $\mathrm{O}$ controle desta doença promoveu adaptações no sistema de manejo dos pomares com a utilização de outros porta-enxertos menos resistentes à seca (BESSANEZI et al., 2003).

A maioria dos citricultores tem dado preferência aos sistemas de irrigação localizada, entre outros motivos, pela economia de água, mas, também, pela facilidade de aplicação de nutrientes via sistema de irrigação, ou seja, fertirrigação. Esta técnica apresenta uma série de vantagens, pois quando os nutrientes são fornecidos juntamente com a água de irrigação, aumenta-se a eficiência da adubação, devido à melhor uniformidade de distribuição e maior possibilidade de parcelamento das adubações e, portanto, mais ajustadas às demandas das plantas (HERNANDEZ, 1994). Há ainda a vantagem de poder ajustar a quantidade aplicada, desde que feito monitoramento adequado.

A programação nutricional adequada na citricultura requer a aferição da disponibilidade de nutrientes através de análises de solo e folha, e também leva em consideração a expectativa de produtividade e a exportação de nutrientes pela colheita. A disponibilidade de $\mathrm{N}$ não tem sido eficientemente avaliada através de análises do solo e, por essa razão, o N-foliar vem sendo empregado como guia para recomendação de nitrogênio em plantas cítricas (QUAGGIO, 2005).

O clorofilômetro, modelo SPAD-502, aparelho desenvolvido pela Minolta no Japão (MINOLTA, 1989), determina indiretamente a concentração de clorofila pela intensidade de coloração verde das folhas. Vários estudos têm demonstrado correlação entre a leitura do aparelho SPAD-502 e o teor de N na folha. O maior benefício desse equipamento é a conveniência e a facilidade de uso, pois as áreas de produção podem ser amostradas e, em poucos minutos, obtém-se a resposta, facilitando assim a tomada de decisão de quanto fertilizante aplicar (PIEKIELEK; FOX, 1992). Peterson et al. (1995) afirmam que o uso do medidor SPAD-502, utilizado como instrumento no manejo da adubação nitrogenada, denominada "adubação quando necessário", é especialmente apropriado, podendo o $\mathrm{N}$ adicional ser aplicado através do sistema de irrigação.

As plantas podem absorver mais $\mathrm{N}$ do que necessitam, a chamada absorção de luxo; porém, para a clorofila, isto não ocorre. A determinação indireta da clorofila atinge um ponto máximo, a partir do qual as leituras são constantes ou com pequenas variações (DWYER, 1995). Alguns autores têm sugerido este ponto de inflexão como indicativo da dose adequada de N (BLACKMER; SCHEPERS, 1995).

Os resultados dos efeitos de diferentes doses e frequência de aplicação de nutrientes via fertirrigação, sobre o estado nutricional e a produtividade das culturas, são divergentes (DIAS, 2003; MONTAG; SHNEK, 2003; DUENHAS et al., 2002). São necessários estudos incluindo técnicas mais eficientes para determinar com maior rapidez e precisão os nutrientes absorvidos e, assim, definir com mais segurança o manejo da adubação via fertirrigação.

Para suprir a falta de informações, outras formas de monitorar a adubação e o estado nutricional das plantas devem ser consideradas. Portanto, o objetivo deste experimento foi avaliar a sensibilidade da medida indireta da clorofila como um método de monitoramento dos níveis de $\mathrm{N}$ em plantas cítricas.

\section{MATERIAL E MÉTODOS}

O experimento foi desenvolvido durante as safras de 2007/2008 e 2008/2009, na Fazenda Emu, pertencente à empresa Citrovita, localizada no Município de Reginópolis, Estado de São Paulo. O objetivo foi instalar o projeto em uma área onde estivessem próximas duas variedades de laranja, assim estas ficariam em um mesmo setor de fertirrigação, para se evitarem outros efeitos de variação. Portanto, foram escolhidas as variedades de copa Valência e Hamlin, ambas sobre o porta-enxerto citrumelo Swingle, por estarem dentro de uma área com as características desejadas para a instalação do projeto e por serem de grande importância para a citricultura paulista. $O$ espaçamento de plantio foi de 7 x 4 m e, nas safras de 2007/2008 e 2008/2009, as plantas possuíam, respectivamente, cinco e seis anos de idade.

Antes do início do projeto, o solo, em toda a área do projeto, possuía as seguintes características químicas, segundo os métodos de análise descritos em Raij et al. (2001), a $0-20 \mathrm{~cm}$ de profundidade: 5,0 de $\mathrm{pH}\left(\mathrm{CaCl}_{2}\right) ; 9 \mathrm{~g} \mathrm{dm}^{-3}$ de $\mathrm{MO} ; 57 \mathrm{mg} \mathrm{dm}^{-3}$ de $\mathrm{P}$ (resina); 21; 3; 14; 5; 22 e 44 mmol $_{c} \mathrm{dm}^{-3} \mathrm{de} \mathrm{H}+\mathrm{Al}$, $\mathrm{K}, \mathrm{Ca}, \mathrm{Mg}, \mathrm{SB}, \mathrm{CTC}$, respectivamente, e $51 \%$ de saturação por bases. A textura do solo na profundidade de $0-60 \mathrm{~cm}$ era arenosa, com teor de argila inferior a $15 \%$, segundo a análise granulométrica do solo realizada conforme descrito por Camargo et al. (1986). O solo foi classificado como Argissolo 
Vermelho-Amarelo (EMBRAPA, 1999).

Os tratamentos foram constituídos por cinco doses de $\mathrm{N}$, aplicadas na forma de nitrato de amônio, via fertirrigação. A dose completa de N (100\%) foi definida com base no histórico de análises de solo e folha, obtidos na fazenda, e também em função da produtividade esperada do talhão, e correspondeu a $140 \mathrm{~kg} \mathrm{ha}^{-1} \mathrm{ano}^{-1}$ (QUAGGIO, 2005). Portanto, foram estabelecidos os seguintes tratamentos: T1 apenas irrigado sem N; T2 fertirrigado com $25 \%$ da dose de $\mathrm{N}$; T3 fertirrigado com $50 \%$ da dose de N; T4 fertirrigado com $100 \%$ da dose de N, e T5 fertirrigado com $200 \%$ da dose de $\mathrm{N}$.

$\mathrm{O}$ parcelamento do $\mathrm{N}$ na fertirrigação foi feito em 34 semanas, desde o início do mês de setembro até o final do mês de abril de cada safra, em duas aplicações semanais, totalizando 68 aplicações. Da dose total indicada em cada tratamento, $25 \%$ desta foi aplicada entre os meses de setembro e outubro, $50 \%$ entre os meses de novembro e março, e os outros $25 \%$ restantes foram aplicados no mês de abril.

As doses de $\mathrm{K}$ e $\mathrm{P}$ também variaram conforme os tratamentos citados. O K, na forma de cloreto de potássio, foi parcelado como o N. O P foi aplicado no início da safra, na forma de ácido fosfórico. Os micronutrientes B, Mn e Zn também foram aplicados via fertirrigação, nas formas de ácido bórico, sulfato de manganês e sulfato de zinco, e o parcelamento seguiu o mesmo do $\mathrm{N}$, porém em doses iguais para todos os tratamentos.

As parcelas foram constituídas por três linhas com doze plantas, sendo que as dez plantas da linha central foram designadas úteis para as avaliações experimentais. As duas linhas laterais e as duas plantas nos extremos da linha central formaram a bordadura. Todo o experimento ocupava área de aproximadamente 4 ha. $\mathrm{O}$ delineamento estatístico empregado foi o de blocos ao acaso, com quatro repetições.

Quanto ao sistema de irrigação, as parcelas possuíam linhas simples de tubo gotejador, instaladas ao lado do tronco das árvores, paralelamente à linha de plantio. O emissor utilizado foi do tipo RAM (Netafim), com vazão de $2,3 \mathrm{~L} \mathrm{~h}^{-1}$ e espaçamento entre emissores de $0,6 \mathrm{~m}$. O bulbo úmido atingia aproximadamente $0,8 \mathrm{~m}$ de largura de faixa molhada. A injeção dos fertilizantes foi feita através do injetor Venturi. O manejo da irrigação foi feito com base na medida diária da evaporação, através do tanque Classe A, obtendo-se a evapotranspiração potencial (ETP) e a evapotranspiração da cultura (ETc), conforme Allen et al. (1998). O experimento também foi monitorado por seis baterias de tensiômetros, e cada bateria possuía três tensiômetros nas profundidades de 30; 60 e $90 \mathrm{~cm}$.
Para a coleta das folhas, foram retiradas quatro folhas por planta, das 10 plantas úteis na parcela, sendo estas a $3^{\mathrm{a}}$ e a $4^{\mathrm{a}}$ folhas recém-maduras, a partir de um dreno (fruto ou brotação nova), nos quatro quadrantes e na altura mediana da planta, totalizando 40 folhas por parcela. As folhas coletadas foram acondicionadas em sacos de papel e levadas ao laboratório para análise do teor de $\mathrm{N}$, conforme a metodologia descrita por Bataglia et al. (1983).

As mesmas folhas coletadas para análise foliar foram utilizadas na determinação da medida indireta da clorofila, realizada utilizando o medidor portátil clorofilômetro, modelo SPAD-502 (MINOLTA, 1989). Este equipamento foi utilizado mediante calibração prévia de acordo com as recomendações do fabricante. As leituras eram feitas no máximo 24 horas após a coleta das folhas, em apenas um lado da nervura, aproximadamente no centro e a $0,6 \mathrm{~mm}$ da margem da lâmina da folha (distância fixada pelo regulador de profundidade do aparelho). O período de 24 horas e a realização da leitura em apenas um lado da nervura foram definidos por um teste realizado com o objetivo de identificar variações da medida indireta da clorofila em um pomar de citros homogêneo e fertirrigado (SOUZA et al., 2007).

As coletas foram realizadas mensalmente, no período de setembro de 2007 a agosto de 2009, safras de 2007/2008 e 2008/2009, sendo feitas 22 coletas de folha e a determinação da medida indireta da clorofila, exceto nos meses de outubro de 2007 e fevereiro de 2009.

Também foram definidos valores de medida indireta da clorofila de acordo com os estádios de desenvolvimento e a probabilidade de resposta esperada à fertirrigação nitrogenada na laranjeira, conforme Godoy et al. (2008) e Silveira et al. (2003). Altas e baixas respostas esperadas corresponderam, respectivamente, aos tratamentos $\mathrm{T} 1, \mathrm{~T} 2$ e $\mathrm{T} 3$ (sem N, 25 e $50 \%$ da dose de N) e T4 e T5 (100 e 200\% da dose de N). Foi considerado, em cada fase do ciclo, o menor valor médio de leitura SPAD (Soil and Plant Analysis Development - unidade adotada pela MINOLTA) observado para as duas variedades.

Os dados obtidos foram submetidos à análise de variância e de regressão linear e polinomial, e a testes de correlação. As médias dos resultados foram comparadas por mês através do teste de Tukey, a $5 \%$ de probabilidade. Toda a estatística foi realizada utilizando o programa SISVAR (FERREIRA, 2003). 


\section{RESULTADOS E DISCUSSÃO}

As variedades de copa estudadas apresentaram comportamento semelhante quanto aos teores de $\mathrm{N}$ foliares e aos valores de leituras SPAD, em relação às doses de $\mathrm{N}$ aplicadas. Por essa razão, a interpretação dos resultados foi realizada através da análise estatística conjunta das safras de 2007/2008 e 2008/2009.

Os valores médios dos 22 meses de determinação da medida indireta da clorofila, assim como do teor de $\mathrm{N}$ foliar, apresentaram ajuste quadrático em função das doses de $\mathrm{N}$ aplicadas via fertirrigação, independentemente da variedade de laranja estudada. Na função ajustada, o valor máximo de leitura SPAD foi de 78, que correspondeu a $245 \mathrm{~kg}$ ha $^{-1}$ ano $^{-1}$ de N (Figura 1).

Foi observada alta correlação entre as médias de cada safra das leituras do medidor de clorofila com as médias correspondentes à concentração foliar de $\mathrm{N}$, com valor de $\mathrm{R}^{2}$ igual a $0,95^{* *}$ (Figura 2). Essa correlação positiva entre o teor de clorofila da folha e o teor de $\mathrm{N}$ foliar é atribuída, principalmente, ao fato de 50 a $70 \%$ do $\mathrm{N}$ total da folha serem integrantes de enzimas (CHAPMAN; BARRETO, 1997) que estão associadas ao cloroplasto (STOCKING; ONGUN, 1962).

Vários autores observaram relação direta entre os valores da medida indireta da clorofila com a concentração de $\mathrm{N}$ nas folhas, em distintas culturas, como café (GODOY et al., 2008), capim-marandu (COSTA et al., 2008), alho (BACKES et al., 2008), milho (GODOY et al., 2007), pimentão (GODOY et al., 2003), feijão (SILVEIRA et al., 2003), batata (GIL et al., 2002), cereais (ARGENTA et al., 2001), tomate (GUIMARÃES et al., 1999), manga, goiaba, tangerina e uva (SHAAHAN et al., 1999), e outras.

Alguns trabalhos, realizados com plantas cítricas também demonstraram a sensibilidade do aparelho SPAD-502. Jifon et al. (2005) estudaram a relação entre a medida indireta da clorofila, obtida por três aparelhos (SPAD-502/Minolta, CCM-200/ Opti-Sciences e Observer/Spectrum Technologies), com a concentração de clorofila e nitrogênio das folhas de seis variedades de citros (Valência, Hamlin, Temple, Volkamer, Redblush e Smooth Flat Seville). As leituras obtidas com o equipamento SPAD-502 apresentaram maiores valores de correlação, respectivamente: 0,97 e 0,54 , para o teor de clorofila e $\mathrm{N}$ foliar, em relação aos outros aparelhos utilizados, considerando os valores obtidos em todas as variedades de citros estudadas. Jesus e Marenco (2008) também observaram correlação de 0,80 entre o teor absoluto de clorofila e a medida indireta da clorofila, obtida pelo SPAD-502, na cultura do limão. Apesar de a leitura SPAD e de a concentração de $\mathrm{N}$ foliar terem apresentado respostas muito semelhante às doses de $\mathrm{N}$ aplicadas (Figura 1), devem-se considerar as diversas vantagens da medida indireta da clorofila em relação à diagnose foliar. Com o auxílio do clorofilômetro, a leitura do estado nutricional da planta pode ser realizada em poucos minutos, possibilitando rápido diagnóstico da situação da lavoura; o aparelho tem custos mínimos de manutenção, ao contrário de outros testes que exigem a compra sistemática de produtos químicos (PIEKIELEK; FOX, 1992); não existe a necessidade de envio de amostras para laboratório, com economia de tempo e dinheiro, e o agricultor pode realizar quantas amostras desejar, sem destruição das folhas (MALAVOLTA et al., 1997).

A amostragem de folhas para análise foliar na cultura da laranja é feita coletando-se a $3^{\text {a }}$ e $4^{a}$ folhas, a partir do fruto, geradas na primavera, com aproximadamente seis meses de idade, normalmente no mês de março e em ramos com frutos de 2 a $4 \mathrm{~cm}$ de diâmetro. Nesta época do ano, o valor adequado de $\mathrm{N}$ na folha deve variar de 23 a $27 \mathrm{~g} \mathrm{~kg}^{-1}$ (QUAGGIO et al., 2005). De acordo com a equação linear obtida entre a leitura SPAD e o teor de N foliar, no mês de março, considerando as duas safras e as variedades ( $\mathrm{y}=$ $1,6 \mathrm{x}+33,2 \mathrm{com} \mathrm{R}^{2}=0,84^{* *}$, semelhante à Figura 2), foi calculado o valor adequado da Leitura SPAD, no período recomendado de coleta das folhas de laranja para análise foliar, que variou de 70,0 a 76,4

Como as doses de $\mathrm{P}$ e $\mathrm{K}$ também variaram conforme os tratamentos, poderiam surgir dúvidas quanto ao efeito isolado de cada nutriente e o desenvolvimento vegetativo das plantas (efeitos de diluição). Porém, após as duas safras, o volume da copa, calculado de acordo com Mendel (1956), não apresentou diferença significativa entre os tratamentos, para as duas variedades de laranja estudadas, sendo que o valor médio observado foi de 25,3 e $27,6 \mathrm{~m}^{3}$, respectivamente, para as laranjeiras Valência e Hamlin.

\section{Variação da leitura SPAD quanto aos estádios de desenvolvimento da cultura}

A medida indireta da clorofila variou de forma significativa em relação às doses de $\mathrm{N}$ aplicadas via fertirrigação, exceto nos meses de setembro, novembro, dezembro, janeiro, março e maio, para a variedade de copa Valência (Tabela 1), e novembro e maio para a variedade de copa Hamlin (Tabela 2). A leitura SPAD exibiu, na maioria dos meses, efeito de ajuste linear em relação às doses de nitrogênio aplicadas, com altos valores de $\mathrm{R}^{2}$, que ficou abaixo de 0,70 , somente nos mês de setembro, para a variedade 
de copa Valência.

Em todos os meses de medida, a leitura SPAD apresentou baixo coeficiente de variação, e isso mostra precisão nesta determinação, sendo que os valores de coeficiente de variação foram, na maioria das vezes, mais baixos que os observados para o teor de $\mathrm{N}$ nas folhas, em ambas as variedades de laranja (Tabelas 1 e 2).

$\mathrm{O}$ teor de $\mathrm{N}$ foliar variou de forma significativa em relação às doses de $\mathrm{N}$ aplicadas via fertirrigação, em quase todos os meses avaliados. A análise de variância só não foi significativa para os meses de dezembro, fevereiro e junho na variedade de copa Valência (Tabela 1) e maio para a laranja Hamlin (Tabela 2). A concentração de $\mathrm{N}$ na folha sempre apresentou ajuste linear em relação aos tratamentos, com valores de $\mathrm{R}^{2}$ altos, superiores a 0,70 exceto no mês de setembro para a variedade de copa Valência.

Entre os meses de setembro e outubro, as plantas de laranja encontram-se em pleno florescimento, ocorre a antese e tem início a formação dos frutos e é também o momento de maior crescimento de ramos vegetativos (MEDINA et al., 2005). Neste período, o aumento em tamanho do fruto é pequeno, mas a atividade metabólica é intensa; nesta fase, formam-se todas as células do fruto, seu tamanho final é resultado do crescimento dessas células (DAVIES; ALBRIGO, 1994). Também tem início a diferenciação de tecidos (exocarpo, mesocarpo e endocarpo), esta fase dura de 1 a 1,5 mês e estende-se até o período de queda fisiológica dos frutos (queda de chumbinho), em novembro/dezembro (MEDINA et al., 2005).

Altos valores de leitura SPAD foram observados em setembro e ocorreu queda nestes valores até o mês de novembro. O mesmo foi observado para o teor foliar de $\mathrm{N}$ na variedade de Valência (Tabela 1). Para a variedade de copa Hamlin, entre os meses de setembro e novembro, a concentração de $\mathrm{N}$ nas folhas mostrou pouca alteração de acordo com o teste de Tukey, a 5\% de probabilidade (Tabela 2 ).

Nos meses de dezembro, janeiro e fevereiro, o fruto encontra-se em crescimento, aumenta a demanda por carboidratos e nutrientes; durante esta fase, o volume celular pode aumentar até mil vezes (DAVIES; ALBRIGO, 1994). Neste período, os valores de leitura SPAD aumentaram em relação ao mês de novembro, o mesmo foi observado para o teor de nitrogênio foliar em ambas as variedades de copa de laranja (Tabelas 1 e 2).

De março a junho, ocorre o período de desenvolvimento dos frutos que leva à maturação fisiológica para a variedade de copa Hamlin de ciclo precoce, enquanto esse período é mais longo de março a setembro, para a variedade de copa Valên- cia de ciclo tardio. Durante a maturação do fruto, o crescimento do endocarpo é pequeno, pois predominam transformações metabólicas que resultam em aumento no conteúdo de sólidos solúveis totais, redução na acidez total do suco e pigmentação da casca (REUTHER, 1977). Para a variedade de copa Hamlin, os valores de leitura SPAD são constantes no período de maturação (Tabela 2). Na variedade de copa Valência, no início da maturação, os valores são baixos, passam por um período em que ficam constates e maiores, entre os meses de abril e junho, e aumentam até o mês de agosto (Tabela 1). O teor de $\mathrm{N}$ foliar, em ambas as variedades, apresentou queda no mês de março e aumento consecutivo até o mês de julho.

Entre os meses de maio e julho, ocorre o período de estresse (indução floral), em que as plantas não foram fertirrigadas e, em agosto, teve início o florescimento da próxima safra. Em julho, foi observado o maior valor de Leitura SPAD para a variedade de copa Hamlin $(79,5)$, e os maiores teores de $\mathrm{N}$ na folha, em ambas as variedades (29 e $30 \mathrm{~g} \mathrm{~kg}^{-1}$, para Valência e Hamlin, respectivamente). Para a variedade de copa Valência, o maior valor de Leitura SPAD ocorreu em setembro $(80,5)$. Os menores valores de medida indireta da clorofila e da concentração de $\mathrm{N}$ foliar, em ambas as variedades, ocorreram no mês de novembro.

Para orientar os produtores quanto à necessidade da adubação nitrogenada, foram estabelecidos valores de leitura SPAD, conforme os estádios de desenvolvimento da cultura da laranja e a probabilidade de resposta à fertirrigação nitrogenada, de acordo com Godoy et al. (2008) e Silveira et al. (2003). Leituras SPAD abaixo de 70 e acima de 75 estiveram relacionadas, respectivamente, com plantas muito responsivas e não responsivas a nitrogênio (Tabela 3), enquanto plantas com leituras intermediárias devem ser adubadas com doses de $\mathrm{N}$ próximas daquelas extraídas com a colheita. Utilizando os mesmos critérios da análise foliar, de acordo com Quaggio et al. (2005), em plantas muito responsivas, aplicar-se-ia de 20 a $25 \%$ a mais da dose recomendada de $\mathrm{N}$ e para plantas pouco responsivas de 20 a $25 \%$ a menos.

Outro indicativo para a tomada de decisão sobre a necessidade da adubação nitrogenada seria através do índice de suficiência de nitrogênio (ISN). Segundo Piekielek et al. (1992), os valores de medida indireta da clorofila podem variar com o manejo, o espaçamento, a variedade, entre outros fatores; portanto, para amenizar estas diferenças, o autor sugere a utilização ISN. O ISN é calculado pela relação entre leitura SPAD obtida em uma parcela, e 
a leitura SPAD obtida em outra parcela, que recebeu alta dose de N, sendo esta a referência. Quando o ISN for menor que 0,95 , é necessária aplicação de $\mathrm{N}$, porém não se leva em consideração a quantidade de $\mathrm{N}$ a ser aplicada.

Neste experimento, os valores de referência para o calculo do ISN foram as leituras SPAD obtidas no tratamento $\mathrm{T} 5$, que recebeu a maior dose de $\mathrm{N}$ (200\% da dose de N), para ambas as variedades. De acordo com a Figura 3, os valores de ISN nas plantas que não foram adubadas (T1) ficaram, na maior parte do ciclo, abaixo de 0,95 , indicando deficiência de N. Para o tratamento T2 ( $25 \%$ da dose de N), os valores de ISN ficaram sempre muito próximos a $0,95 \mathrm{e}$, em alguns momentos, abaixo, nos meses de outubro, janeiro e março. Os outros tratamentos (T3 e T4, respectivamente, 50 e $100 \%$ da dose de N) apresentaram ISN sempre maior que 0,95 durante todo o ciclo de desenvolvimento da cultura.

A correlação entre a medida indireta da clorofila e o teor de $\mathrm{N}$ na folha sempre foi alta em todos os meses de determinação e em ambas as variedades de copa estudadas. A medida indireta da clorofila apresentou amplitude igual ou maior na variação dos dados em relação ao teor de nitrogênio foliar, refletindo melhor a variação das doses de nitrogênio aplicadas, provavelmente pela maior sensibilidade do aparelho em relação à concentração foliar de $\mathrm{N}$. Considerando as médias observadas durante os meses para as duas variedades, a leitura SPAD variou de 69,2 a 80,5 (11 unidades), e o teor de $\mathrm{N}$ foliar variou de 25 a $30 \mathrm{~g} \mathrm{~kg}^{-1}$ (5 unidades), conforme as Tabelas 1 e 2 .

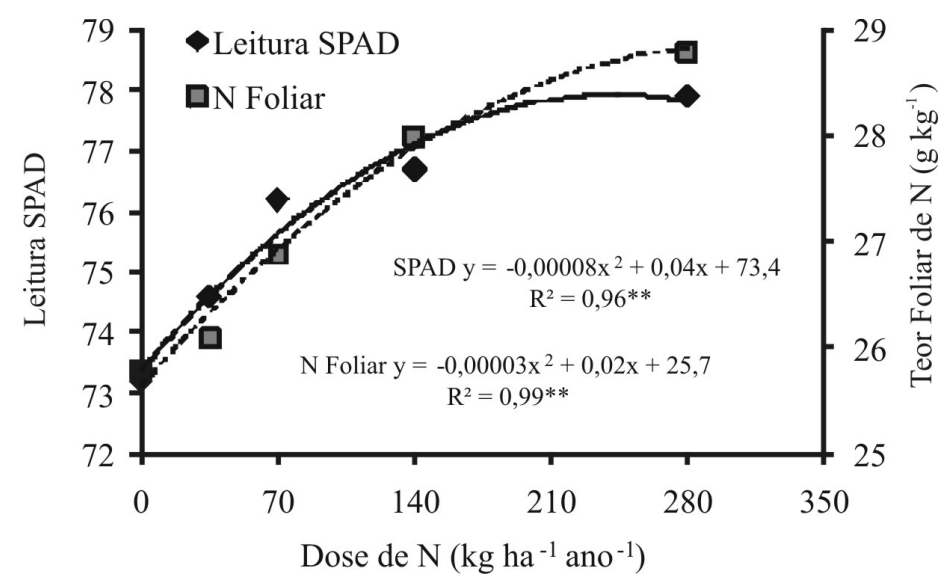

FIGURA 1 - Valores de leitura SPAD e teor de nitrogênio na folha, em relação a doses de nitrogênio aplicadas (valores médios observados para as duas variedades de laranja).

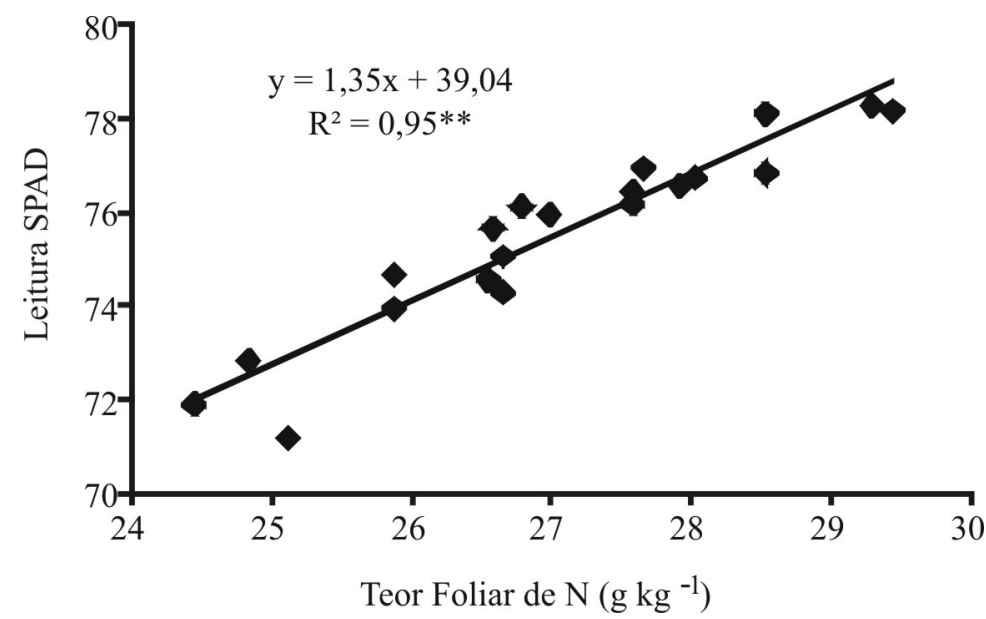

FIGURA 2 - Correlação entre a leitura SPAD e o teor de N nas folhas das laranjeiras Valência e Hamlin (valores médios obtidos em cada variedade e safra avaliada). 


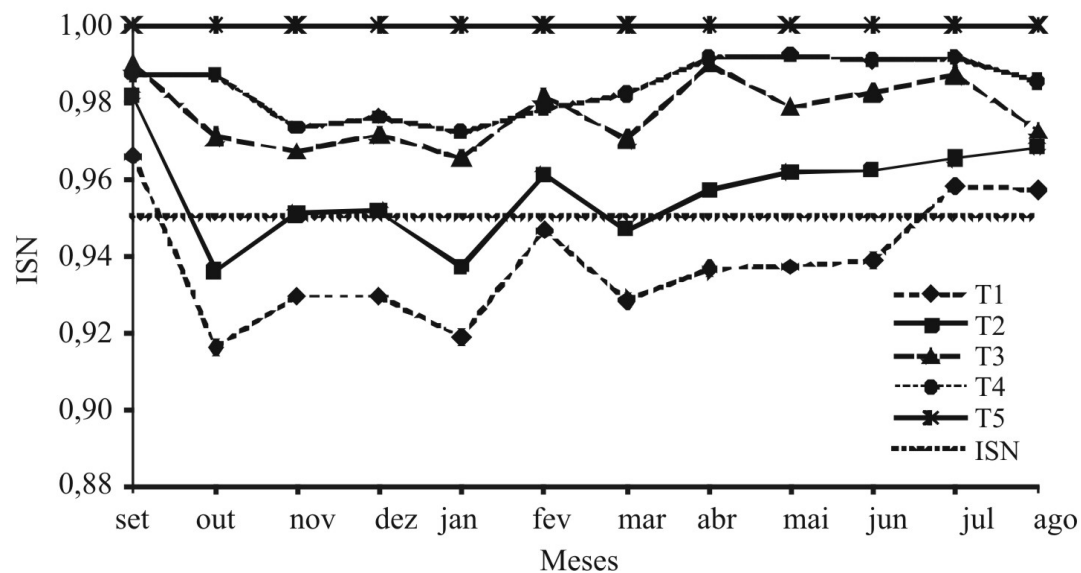

FIGURA 3 - Índice de suficiência de nitrogênio (ISN) de acordo com os estádios de desenvolvimento da cultura (valores médios observados para as duas variedades de laranja).

TABELA 1 - Resultados de medida indireta da clorofila e teor de nitrogênio na folha, em função das doses de nitrogênio aplicadas, para a variedade de copa Valência, no período de setembro de 2007 a agosto de 2008 (médias mensais correspondentes às duas safras).

\begin{tabular}{|c|c|c|c|c|c|c|c|c|c|c|c|c|}
\hline \multirow{2}{*}{$\begin{array}{l}\text { Dose de N } \\
\left(\mathrm{kg} \mathrm{ha}^{-1}\right) \\
\end{array}$} & \multicolumn{12}{|c|}{ Meses de coleta das folhas (estádio fenológico) } \\
\hline & $\begin{array}{l}\text { Set. } \\
\text { (a) }\end{array}$ & $\begin{array}{c}\text { Out. } \\
\text { (a) }\end{array}$ & $\begin{array}{l}\text { Nov. } \\
\text { (ab) }\end{array}$ & $\begin{array}{c}\text { Dez. } \\
\text { (b) }\end{array}$ & $\begin{array}{c}\text { Jan. } \\
\text { (b) }\end{array}$ & $\begin{array}{c}\text { Fev. } \\
\text { (b) }\end{array}$ & $\begin{array}{r}\text { Mar. } \\
\text { (bc) }\end{array}$ & $\begin{array}{c}\text { Abr. } \\
\text { (c) }\end{array}$ & $\begin{array}{l}\text { Maio } \\
\text { (cd) }\end{array}$ & $\begin{array}{l}\text { Jun. } \\
\text { (cd) }\end{array}$ & $\begin{array}{l}\text { Jul. } \\
\text { (cd) }\end{array}$ & $\begin{array}{r}\text { Ago. } \\
\text { (da) }\end{array}$ \\
\hline & \multicolumn{12}{|c|}{ Leitura SPAD $^{(1)}$} \\
\hline 0 & 79,2 & 72,0 & 66,0 & 74,5 & 70,9 & 76,7 & 72,9 & 73,5 & 72,5 & 73,3 & 74,2 & 75,5 \\
\hline 35 & 80,4 & 71,8 & 68,3 & 76,1 & 71,9 & 78,6 & 73,1 & 74,5 & 74,8 & 74,7 & 74,5 & 76,8 \\
\hline 70 & 80,9 & 74,4 & 70,1 & 77,4 & 73,8 & 80,0 & 73,8 & 77,2 & 76,1 & 76,6 & 77,3 & 77,6 \\
\hline 140 & 80,8 & 75,4 & 69,8 & 77,0 & 74,8 & 79,5 & 75,2 & 77,6 & 77,4 & 77,6 & 78,0 & 77,9 \\
\hline 280 & 81,3 & 75,9 & 72,0 & 78,6 & 76,5 & 81,2 & 76,5 & 76,9 & 77,7 & 78,1 & 77,9 & 78,8 \\
\hline
\end{tabular}

Médias $\quad 80,5$ a 73,9 ef $69,2 \mathrm{~g} 76,7$ bcd 73,6 f 79,2 ab74,3 def 75,9 cdef 75,7 cdef 76,0 cdef76,3 cde 77,3 bc

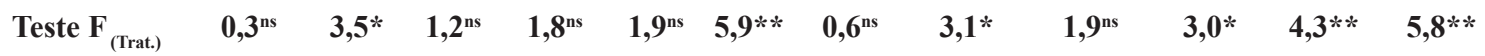

\begin{tabular}{lcccccccccccc}
$\mathrm{CV}(\%)$ & 4,9 & $\mathbf{2 , 8}$ & $\mathbf{8 , 4}$ & $\mathbf{4 , 2}$ & $\mathbf{6 , 2}$ & $\mathbf{1 , 7}$ & $\mathbf{7 , 5}$ & $\mathbf{3 , 8}$ & $\mathbf{5 , 7}$ & $\mathbf{4 , 3}$ & $\mathbf{3 , 4}$ & $\mathbf{1 , 8}$ \\
\hline $\mathrm{R}^{2}$ & $\mathbf{0 , 6 4}$ & $\mathbf{0 , 7 4}$ & $\mathbf{0 , 8 0}$ & $\mathbf{0 , 7 4}$ & $\mathbf{0 , 9 1}$ & $\mathbf{0 , 7 3}$ & $\mathbf{0 , 9 7}$ & $\mathbf{0 , 9 2}$ & $\mathbf{0 , 7 0}$ & $\mathbf{0 , 7 6}$ & $\mathbf{0 , 9 1}$ & $\mathbf{0 , 8 2}$
\end{tabular}

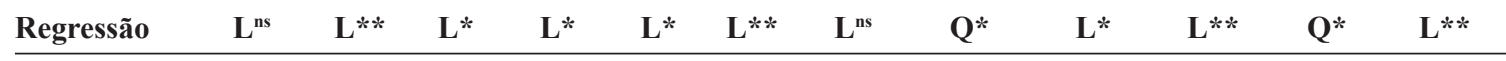

\begin{tabular}{llllllllllllll}
\hline \multicolumn{10}{c}{ Teor foliar de N $\mathbf{~ ( g ~ k g - 1 ) ~}$} \\
0 & 25 & 23 & 23 & 25 & 24 & 27 & 25 & 26 & 26 & 28 & 29 & 26 \\
35 & 26 & 25 & 24 & 25 & 25 & 27 & 25 & 25 & 26 & 28 & 28 & 27 \\
70 & 27 & 25 & 25 & 26 & 26 & 27 & 26 & 27 & 27 & 28 & 29 & 27 \\
140 & 27 & 26 & 26 & 28 & 27 & 29 & 27 & 29 & 28 & 29 & 30 & 28 \\
280 & 27 & 26 & 26 & 28 & 27 & 29 & 28 & 29 & 29 & 29 & 31 & 29 \\
\hline
\end{tabular}

Médias 26,4 def 25,0 gh 24,7 h 26,6 def 26,0 fg27,8 be 26,2 ef 26,9 cdef 27,4 bed 28,3 ab 29,3 a 27,2 bcde

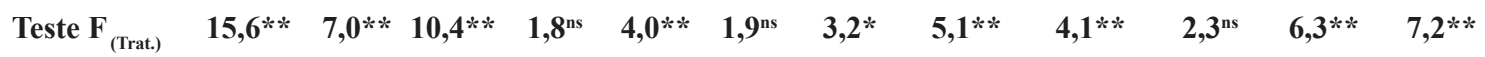

$\begin{array}{lcccccccccccc}\text { CV (\%) } & \mathbf{2 , 5} & \mathbf{3 , 5} & \mathbf{4 , 3} & \mathbf{9 , 7} & \mathbf{7 , 5} & \mathbf{5 , 1} & \mathbf{8 , 5} & \mathbf{7 , 1} & \mathbf{6 , 6} & \mathbf{5 , 1} & \mathbf{4 , 2} & \mathbf{3 , 9} \\ \mathbf{R}^{2} & \mathbf{0 , 9 7} & \mathbf{0 , 8 2} & \mathbf{0 , 9 2} & \mathbf{0 , 8 4} & \mathbf{0 , 7 8} & \mathbf{0 , 8 1} & \mathbf{0 , 8 8} & \mathbf{0 , 7 8} & \mathbf{0 , 9 6} & \mathbf{0 , 7 4} & \mathbf{0 , 8 2} & \mathbf{0 , 9 3} \\ \text { Regressão }^{*} & \mathbf{Q}^{* *} & \mathbf{L}^{* *} & \mathbf{L}^{* *} & \mathbf{L}^{*} & \mathbf{L}^{* *} & \mathbf{L}^{*} & \mathbf{L}^{* *} & \mathbf{L}^{* *} & \mathbf{L}^{* *} & \mathbf{L}^{*} & \mathbf{L}^{* *} & \mathbf{L}^{* *} \\ \text { Correlação }^{(2)} & \mathbf{0 , 9 6} & \mathbf{0 , 9 6} & \mathbf{0 , 9 2} & \mathbf{0 , 7 7} & \mathbf{0 , 9 6} & \mathbf{0 , 6 2} & \mathbf{0 , 9 9} & \mathbf{0 , 8 1} & \mathbf{0 , 8 8} & \mathbf{0 , 8 1} & \mathbf{0 , 7 6} & \mathbf{0 , 9 4}\end{array}$

(ग) Leitura SPAD (Soil and Plant Analysis Development - unidade adotada pela MINOLTA). (a) florescimento, antese e formação do fruto (ab) queda de chumbinho (b) crescimento do fruto, (bc) final crescimento e início maturação, (c) maturação, (cd) maturação e período de estresse (da) final maturação e início florescimento. Médias seguidas por letras iguais na horizontal não diferem entre si, pelo teste de Tukey, a 5\% de probabilidade. **- significativo a $1 \%,{ }^{*}$ - significativo a $5 \%$, ns - não significativo, Trat. - Tratamento, L ajuste linear, Q - ajuste quadrático, C. V. - coeficiente de variação e ${ }^{(2)}$ correlação entre a Leitura SPAD e o teor foliar de N. 
TABELA 2 - Resultados de medida indireta da clorofila e teor de nitrogênio na folha, em função das doses de nitrogênio aplicadas, para a variedade de copa Hamlin, no período de setembro de 2007 a agosto de 2009 (médias mensais correspondentes às duas safras).

\begin{tabular}{|c|c|c|c|c|c|c|c|c|c|c|c|c|}
\hline \multirow{2}{*}{$\begin{array}{l}\text { Dose de N } \\
\left(\mathrm{kg} \mathrm{ha}^{-1}\right) \\
\end{array}$} & \multicolumn{12}{|c|}{ Meses de coleta das folhas (estádio fenológico) } \\
\hline & $\begin{array}{l}\text { Set. } \\
\text { (a) }\end{array}$ & $\begin{array}{l}\text { Out. } \\
\text { (a) }\end{array}$ & $\begin{array}{l}\text { Nov. } \\
\text { (ab) }\end{array}$ & $\begin{array}{c}\text { Dez. } \\
\text { (b) }\end{array}$ & $\begin{array}{c}\text { Jan. } \\
\text { (b) }\end{array}$ & $\begin{array}{c}\text { Fev. } \\
\text { (b) }\end{array}$ & $\begin{array}{l}\text { Mar. } \\
\text { (bc) }\end{array}$ & $\begin{array}{l}\text { Abr. } \\
\text { (c) }\end{array}$ & $\begin{array}{l}\text { Maio } \\
\text { (cd) }\end{array}$ & $\begin{array}{l}\text { Jun. } \\
\text { (cd) }\end{array}$ & $\begin{array}{l}\text { Jul. } \\
\text { (d) }\end{array}$ & $\begin{array}{l}\text { Ago. } \\
\text { (da) }\end{array}$ \\
\hline & \multicolumn{12}{|c|}{ Leitura SPAD $^{(1)}$} \\
\hline 0 & 75,8 & 66,5 & 68,2 & 69,9 & 72,3 & 74,4 & 71,9 & 72,4 & 74,3 & 74,0 & 78,2 & 76,8 \\
\hline 35 & 77,1 & 69,7 & 69,0 & 71,8 & 74,2 & 74,8 & 74,6 & 74,6 & 75,8 & 76,3 & 79,0 & 77,3 \\
\hline 70 & 77,9 & 72,4 & 69,6 & 73,6 & 76,7 & 76,7 & 77,6 & 77,1 & 77,2 & 77,6 & 79,7 & 77,2 \\
\hline 140 & 77,6 & 73,9 & 70,8 & 74,7 & 76,8 & 76,7 & 78,0 & 76,9 & 78,0 & 77,9 & 79,7 & 78,9 \\
\hline 280 & 79,1 & 75,3 & 72,4 & 76,8 & 79,4 & 78,4 & 79,5 & 78,9 & 78,9 & 78,8 & 81,1 & 80,3 \\
\hline Médias & 77,5 abc & $71,6 \mathrm{de}$ & $70,0 \mathrm{e}$ & $73,4 \mathrm{~d}$ & $75,9 \mathrm{c}$ & 76,2 bc & $76,3 \mathrm{bc}$ & 76,0 bc & 76,9 bc & 76,9 bc & 79,5 a & 78,1 ab \\
\hline Teste $\mathbf{F}_{\text {(Trat) }}$ & $3,1^{*}$ & $29,1^{* *}$ & $1,2^{\mathrm{ns}}$ & $2,9 *$ & $8,4^{* * *}$ & $3,0^{*}$ & $6,2 * *$ & $5,6 * *$ & $1,9^{\text {ns }}$ & $2,5^{*}$ & $3,2 *$ & $5,5 * *$ \\
\hline C V $(\%)$ & 2,5 & 1,8 & 6,2 & 6,1 & 3,5 & 2,5 & 4,5 & 3,9 & 4,8 & 4,2 & 2,1 & 2,2 \\
\hline $\mathbf{R}^{2}$ & 0,79 & 0,97 & 0,98 & 0,91 & 0,86 & 0,87 & 0,93 & 0,78 & 0,81 & 0,70 & 0,91 & 0,97 \\
\hline \multirow[t]{2}{*}{ Regressão } & $\mathbf{L}^{* *}$ & $Q^{* *}$ & $Q^{*}$ & $L^{* *}$ & $L^{* *}$ & $L^{* *}$ & $Q^{*}$ & $\mathbf{L}^{* *}$ & $\mathbf{L}^{*}$ & $\mathbf{L}^{*}$ & $\mathbf{L}^{* *}$ & $\mathbf{L}^{* *}$ \\
\hline & \multicolumn{12}{|c|}{ Teor foliar de $\mathrm{N}\left(\mathrm{g} \mathrm{kg}^{-1}\right)$} \\
\hline 0 & 24 & 25 & 24 & 26 & 25 & 27 & 26 & 26 & 27 & 28 & 29 & 25 \\
\hline 35 & 25 & 24 & 24 & 26 & 25 & 28 & 26 & 26 & 28 & 29 & 30 & 25 \\
\hline 70 & 25 & 26 & 26 & 28 & 27 & 28 & 27 & 27 & 28 & 29 & 30 & 26 \\
\hline 140 & 26 & 27 & 27 & 28 & 28 & 29 & 28 & 29 & 29 & 30 & 30 & 27 \\
\hline 280 & 28 & 29 & 28 & 30 & 29 & 30 & 29 & 30 & 30 & 31 & 32 & 28 \\
\hline Médias & $25,6 \mathrm{f}$ & 26,3 ef & $25,9 \mathrm{f}$ & $27,6 \mathrm{~cd}$ & 26,7 def & 28,5 bc & 27,0 de & $27,7 \mathrm{~cd}$ & $28,2 \mathrm{c}$ & 29,4 ab & 30,2 a & 26,4 ef \\
\hline Teste $\mathbf{F}_{\text {(Trat.) }}$ & $6,5 * *$ & $38,4 * *$ & $12,0 * *$ & $5,9 * *$ & $15,6^{* *}$ & $7,4 * *$ & $6,2 * *$ & $17,9 * *$ & $1,6^{\mathrm{ns}}$ & $7,5^{* *}$ & $8,3^{* *}$ & $12,3^{* *}$ \\
\hline C V $(\%)$ & 5,6 & 2,2 & 4,8 & 7,7 & 5,0 & 3,4 & 6,5 & 3,8 & 7,9 & 4,1 & 3,5 & 4,5 \\
\hline $\mathbf{R}^{2}$ & 0,98 & 0,90 & 0,88 & 0,88 & 0,94 & 0,95 & 0,90 & 0,89 & 0,99 & 0,97 & 0,96 & 0,91 \\
\hline Regressão & $\mathbf{L}^{* *}$ & $L^{* *}$ & $\mathbf{L}^{* *}$ & $\mathbf{L}^{* *}$ & $L^{* *}$ & $\mathbf{L}^{* *}$ & $\mathbf{L}^{* *}$ & $\mathbf{L}^{* *}$ & $L^{*}$ & $\mathbf{L}^{* *}$ & $\mathbf{L}^{* *}$ & $\mathbf{L}^{* *}$ \\
\hline Correlação $^{(2)}$ & 0,91 & 0,83 & 0,95 & 0,95 & 0,95 & 0,91 & 0,89 & 0,85 & 0,94 & 0,90 & 0,95 & 0,95 \\
\hline
\end{tabular}

(1) Leitura SPAD (Soil and Plant Analysis Development - unidade adotada pela MINOLTA). (a) florescimento, antese e início formação do fruto, (ab) queda de chumbinho (b) crescimento do fruto, (bc) final crescimento e início maturação (c) maturação (cd) final da maturação e período de stress (d) stress (da) final de stress e início florescimento. Médias seguidas por letras iguais na horizontal não diferem entre si pelo teste de Tukey a $5 \%$. **- significativo a $1 \%$, * - significativo a $5 \%$, ns - não significativo, L - ajuste linear, Q - ajuste quadrático, C. V. - coeficiente de variação e (2) correlação entre a Leitura SPAD e o teor foliar de N.

TABELA 3 - Faixas de leitura SPAD de acordo com a reposta esperada à fertirrigação nitrogenada em cada estádio de desenvolvimento da cultura (valores médios observados para as duas variedades de laranja).

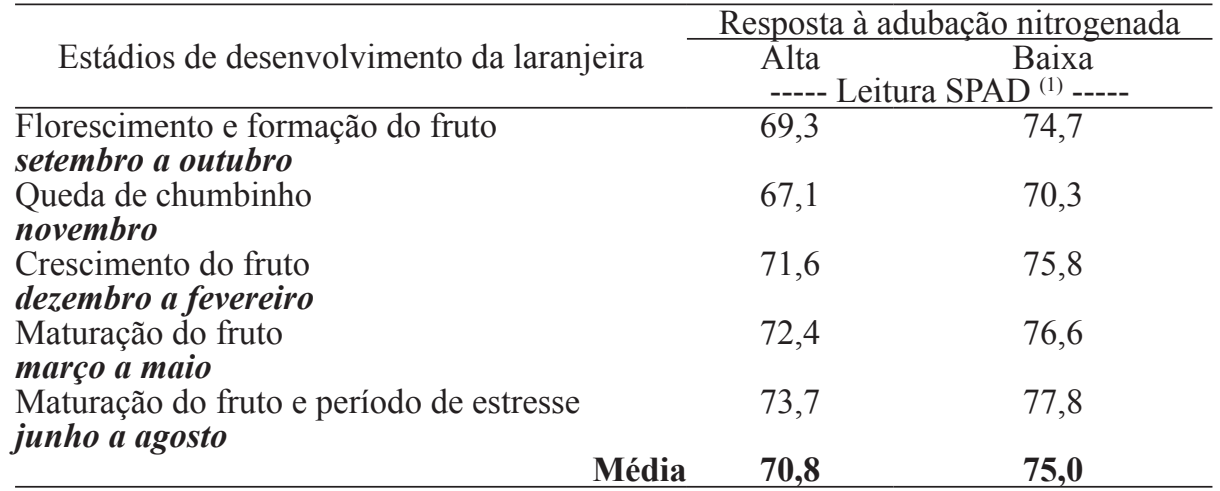

${ }^{(1)}$ Leitura SPAD (Soil and Plant Analysis Development - unidade adotada pela MINOLTA). 


\section{CONCLUSÕES}

1-A medida indireta da clorofila mostra alta correlação com as doses de nitrogênio aplicadas via fertirrigação nas duas variedades de laranja estudadas.

2-A leitura SPAD correlaciona-se linearmente ao teor de $\mathrm{N}$ na folha em todos os meses de determinação.

3-As variedades de laranja estudadas apresentam comportamento semelhante quanto aos valores de leitura SPAD.

4-Leituras SPAD abaixo de 70 e acima de 75 estão relacionadas, respectivamente, com plantas muito responsivas e não responsivas a nitrogênio, enquanto plantas com leituras intermediárias devem ser adubadas com doses de $\mathrm{N}$ próximas daquelas extraídas com a colheita.

5-Baseando-se em duas safras completas, é possível concluir que a medida indireta da clorofila pode ser empregada como ferramenta rápida e não destrutiva no monitoramento e na avaliação da disponibilidade de nitrogênio em plantas cítricas.

\section{AGRADECIMENTOS}

À Fundação de Amparo à Pesquisa do Estado de São Paulo (FAPESP), pela concessão de bolsa e auxílio-pesquisa; ao Instituto Agronômico de Campinas (IAC), pelo apoio durante as análises, e à empresa Citrovita - Votorantim/Agroindústria, pela contribuição e participação no desenvolvimento deste ensaio.

\section{REFERÊNCIAS}

ALLEN, R. G.; PEREIRA, L. S.; RAES, D.; SMITH, M. Crop evapotranspiration: gidelines for computing crop wáter requirements. Roma: FAO, 1998. 300p. (Irrigation and Drainage, Paper 56).

ARGENTA, G.; SILVA, P. R. F.; BORTOLINI, C. G. Clorofila na folha como indicador do nível de nitrogênio em cereais. Ciência Rural, Santa Maria, v.31, n.4, p. 715-722, 2001.
BACKES, C.; LIMA, C. P.; GODOY, L. J. G.; VILLAS BÔAS, R. L.; IMAIZUMI, I. Coloração verde nas folhas na cultura do alho vernalizado em resposta a adubação nitrogenada. Bragantia, Campinas, v.67, n.2, p.491-498, 2008.

BATAGLIA. O. C.; FURLANI, A. M. C.; TEIXEIRA, J. P. F.; FURLANI, P. R.; GALO, J. R. Métodos de análise química de plantas. Campinas: Instituto Agronômico, 1983. 48p. (Circular, 78).

BESSANEZI, R. B.; FERNANDES, N. G.; YAMAMOTO, P. T. Morte súbita dos citros. Araraquara: Fundecitrus, 2003. 54p.

BLACKMER, T. M.; SCHEPERS, J. S. Use of chlorophyll meter to monitor crop nitrogen status and schedulet fertigation for corn. Journal of Production Agriculture, Madison, v.8, p.56-60, 1995.

CAMARGO, O. A.; MONIZ, A. C; JORGE, J. A.; VALADARES, J. M. A. S. Métodos de análise química, mineralógica e física de solos do Instituto Agronômico de Campinas. Campinas: Instituto Agronômico, 1986. 94p. (Boletim Técnico, 106).

CHAPMAN, S.C.; BARRETO, H.J. Using a chlorophyll meter to estimative specific leaf nitrogen of tropical maize during vegetative growth. Agronomy Journal, Madison, v.89, p.557-562, 1997.

COELHO, R. B. Irrigação por gotejo: problemas e soluções. Citricultura Atual, Cordeirópolis, n.76, p.12-14, 2010.

COSTA, K. A. P.; FAQUIM, V.; OLIVEIRA, I. P.; RODRIGUES, R. B. Doses e fontes de nitrogênio em pastagem de capim-marandu. II - nutrição nitrogenada da planta. Revista Brasileira de Ciência do Solo, Campinas, v.32, p.1601-1607, 2008.

DAVIES, W. J.; ALBRIGO, L. G. Citrus. Wallingford: Cab Internacional, 1994. 254p.

DIAS, N. S. Manejo da fertirrigação e controle da salinidade em solo cultivado com melão rendilhado sob ambiente protegido. 2004. $110 \mathrm{f}$. Tese (Doutorado) - Escola Superior de Agricultura Luiz de Queiroz, Universidade de São Paulo, Piracicaba, 2004. (corrigir texto p. 1. de 2003 para 2004)

DUENHAS, L. H.; VILLAS BÔAS, R. L.; SOUZA, C. M. P.; RAGOZO, C. R. A.; BÜLL, L. T. Fertirrigação com diferentes doses de NPK e seus efeitos sobre a produção e qualidade de frutos de laranja (Citrus sinensis) 'Valência'. Revista Brasileira de Fruticultura, Jaboticabal, v.24, n.1, p.214-218, 2002. 
DWYER, L.M. Quantifying the nonlinearity in chlorophyll meter response to corn leaf nitrogen concentration. Journal of Plant Science, Amsterdam, v.75, p.179-182, 1995.

EMBRAPA. Sistema brasileiro de classificação de solos. Rio de Janeiro: Centro Nacional de Pesquisa de Solos, 1999. 412p.

FERREIRA, D. F. Sisvar versão 4.2. Lavras: DEX, UFLA, 2003.

GIL, P. T.; FONTES, P. C. R.; CECON, P. R.; FERREIRA, F. A. Índice SPAD para o diagnóstico do estado de nitrogênio e para o prognóstico da produtividade da batata. Horticultura Brasileira, Campinas, v. 20, p. 611-615, 2002.

GODOY, L. J. G.; FERNANDES, D. M.; SOUTO, L. S.; VILLAS BOAS, R. L. Uso do clorofilômetro no manejo da adubação nitrogenada para milho em sucessão a pastagem de Brachiaria decumbens. Ciência Rural, Santa Maria, v.37, n.1, p.38-44, 2007.

GODOY, L. J. G.; SANTOS, T. S.; VILLAS BÔAS, R. L.; LEITE JÚNIOR, J. B. Índice relativo de clorofila e o estado nutricional de nitrogênio durante o ciclo do cafeeiro fertirrigado. Revista Brasileira de Ciência do Solo, Viçosa, MG, v.32, p. 217-226, 2008.

GODOY, L. J. G.; VILLAS BÔAS, R. L.; BULL, L. T. Utilização da medida do clorofilômetro no manejo da adubação nitrogenada em plantas de pimentão. Revista Brasileira de Ciência do Solo, Viçosa, MG, v.27, p.1.049-1.056, 2003.

GUIMARÃES, T. G.; FONTES, P. C. R.; PEREIRA, P. R. G.; ALVAREZ, V.; MONNERAT, P. H. Teores de clorofila determinados por medidor portátil e sua relação com formas de nitrogênio em folhas de tomateiro cultivados em dois tipos de solo. Bragantia, Campinas, v.58, n.1, p.209-216, 1999.

HERNANDEZ, F. B. T. Potencialidades de fertirrigação, In: VITTI, G. C.; BOARETTO, A. E. (Ed.). Fertilizantes fluídos. Piracicaba: POTAFOS, 1994. p. 215-225.

JESUS, S. V.; MARENCO, R. A. O SPAD-502 como alternativa para a determinação dos teores de clorofila em espécies frutíferas. Acta Amazonica, Manaus, v.38, n.4, p.815-818, 2008.
JIFON, J. L.; SYVERTSEN, J. P.; WHALEY, E. Growth environment and leaf anatomy affect nondestructive estimates of chlorophyll and nitrogen in citrus sp. leaves. Journal of the American Society for Horticultural Science, Alexandria, v.130, n.2, p.152-158, 2005.

MALAVOLTA, E.; VITTI, G. C.; OLIVEIRA, S. A. Avaliação do estado nutricional das plantas: princípios e aplicações. Piracicaba: POTAFÓS, 1997. 319p.

MINOLTA CAMERA Co. Ltd. Manual for chlorophyll meter SPAD-502. Osaka: Minolta Radiometric Instruments, 1989. 22p.

MEDINA, C. L.; RENA, A. B.; MACHADO, E. C.; SIQUEIRA, D. L. Fisiologia dos citros. In: MATTOS JUNIOR, D.; NEGRI, J. R.; PIO, R. M.; POMPEU JUNIOR, J. Citros. Campinas: Instituto Agronômico e Fundag, 2005. p. 147 - 196.

MENDEL, K. Rootstock-scion relationships in Shamouti trees on light soil. Ktavim, Rehovot, v. 6, p. 35-60, 1956

MONTAG, U. J.; SHNEK, M. Principles of fertigation and their potential for global application. Disponível em: $<$ http://www.fertilizer. org/PUBLISH/PUBENV/Fertigb8.htm. . . Acesso em: 16 maio 2003

PETERSON, T.A.; BLACKMER, T.M.; SCHEPERS, J.S. NebGuide: using a chlorophyll meter to improve $\mathrm{N}$ management. Lincoln: University of Nebraska, Cooperative Extension, Institute of Agricultural and Natural Resources, 1995. 5 p.

PIEKIELEK, W. P.; FOX, R. H. Use of a chlorophyll meter to predict sidedress nitrogen requeriments for mayze. Agronomy Journal, Madison, v.84, p.5965, 1992.

QUAGGIO, J. A.; MATTOS JUNIOR, D.; CANTARELLA, H. Manejo da fertilidade do solo na citricultura. In: MATTOS JUNIOR, D.; DE NEGRI., J. D.; PIO, R. M.; POMPEU JUNIOR, J. Citros. Campinas: Instituto Agronômico e Fundag, 2005. p. 483 - 507.

RAIJ, B. V.; ANDRADE, J. C.; CANTARELLA, H.; QUAGGIO, J. A. Análise química para avaliação da fertilidade de solos tropicais. Campinas: Instituto Agronômico, 2001. 285p. 
REWTHER, W. Cítrus. In: ALVIM, P. T.; KOZLOWSKI, T. T. Ecophysiology of tropical crops. New York: Academic Press, 1977. p. 409-439.

SHAAHAN, M. M.; EL-SAYED, A. A.; ABOU ELNOUR, E. A. A. Predicting nitrogen, magnesium and iron nutritional status some perennial crops using a portable chlorophyll meter. Horticultural Science, Ashford, v. 82, p. 339-348, 1999.

SILVEIRA, P. M.; BRAZ, A. J. B. P.; DIDONET, A. D. Uso do clorofilômetro como indicador da necessidade de adubação nitrogenada em cobertura no feijoeiro. Pesquisa Agropecuária Brasileira, Brasília, v.38, n.9, p. 1083-1087, 2003.
SOUZA, T. R.; VILLAS BÔAS, R. L.; FERNANDES, D. M.; BACKES, C.; GODOY, L. J. G. Variação da medida SPAD, determinada pelo clorofilômetro em um pomar de citros fertirrigado. In: CONGRESSO BRASILEIRO DE CIÊNCIA DO SOLO, 31., 2007. Gramado. Anais... CD-ROM.

STOKING, C.R.; ONGUN, A. The intracellular distribution of some metallic elements in leaves. American Journal of Botany, Columbus, v.49, p.284-289, 1962. 